\title{
Es EILD - Anforderungen an die Publikation freier Lerneinheiten (OER) im Fach Datenbanken
}

\author{
Thomas C. Rakow ${ }^{1}$ (D) $\cdot$ Heide Faeskorn-Woyke ${ }^{2} \cdot$ Inga Marina Saatz ${ }^{3} \cdot$ Harm Knolle $^{4}$
}

Eingegangen: 1. Oktober 2020 / Angenommen: 11. Februar 2021 / Online publiziert: 3. März 2021

(c) Der/die Autor(en) 2021

\section{Zusammenfassung}

Kompetenzen auf dem Gebiet der Datenbanken gehören zum Pflichtbereich der Informatik. Das Angebot an Lehrbüchern, Vorlesungsformaten und Tools lässt sich jedoch für Lehrende oft nur eingeschränkt in die eigene Lehre integrieren. In diesem Aufsatz schildern wir unsere Erfahrungen in der Nutzung (frei) verfügbarer und der Entwicklung eigener digitaler Inhalte für grundlegende Datenbankveranstaltungen. Die Präferenzen der Studierenden werden mittels Nutzungsanalysen und Befragungen ermittelt. Wir stellen die Anforderungen auf, wie die nicht selten aufwendig herzustellenden digitalen Materialien von Lehrenden in ihre Lehr- und Lernumgebungen integriert werden können. Als konstruktive Antwort auf diese Herausforderung wird das Konzept EILD zur Entwicklung von Inhalten für die Lehre im Fach Datenbanken vorgestellt. Die Inhalte sollen in vielfältigen Lernszenarien eingesetzt werden können und mit einer Creative Commons (CC) Lizenzierung als OER (open educational resources) frei zur Verfügung stehen.

Schlüsselwörter Datenbanksysteme · Hochschullehre · Digitale Lehre · Open Educational Ressources · OER · Creative Commons $\cdot \mathrm{CC}$

\section{Einleitung}

Digitale Lernformen in der Hochschullehre sind weit verbreitet. Wannemacher et al. werten etwa 250 „Fallstudien und -beispiele digitalisierter Lernelemente und -formate“ aus [39]. In Zeitschriften wie eleed und auf Konferenzen wie der Fachtagung Bildungstechnologien (ehemals: Die e-Learning Fachtagung Informatik, DELFI), der Fachtagung Hochschuldidaktik Informatik und der European Con-

Mitarbeiter der Autoren arbeiten in dem Projekt EILD.nrw, das durch das Land Nordrhein-Westfalen in einem

Kooperationsvorhaben der Digitalen Hochschule NRW (DH.NRW) gefördert wird.

Thomas C. Rakow

thomas.rakow@hs-duesseldorf.de

1 Fachbereich Medien, Hochschule Düsseldorf, Düsseldorf, Deutschland

2 Fakultät für Informatik und Ingenieurwissenschaften, Technische Hochschule Köln, Gummersbach, Deutschland

3 Fachbereich Informatik, Fachhochschule Dortmund, Dortmund, Deutschland

4 Fachbereich Informatik, Hochschule Bonn-Rhein-Sieg, Sankt Augustin, Deutschland ference on Technology Enhancd Learning (EC-TEL) werden Arbeiten zur digitalen Lehre seit vielen Jahren publiziert. In 2019 fordert die Kultusministerkonferenz die Digitalisierung von Studium und Lehre [24]. Der Fakultätentag Informatik sieht die Herausforderung in den notwendigen Investitionen [12].

Trotz der Menge an Inhalten herrscht aber große Ratlosigkeit, ob und wie diese Angebote in die Curricula an den Hochschulen eingebunden werden können. Auf der Habenseite digitaler Formate stehen die immer und überall vorhandene Verfügbarkeit, die Individualisierbarkeit, insbesondere mit einem individuellen Tempo des Rezipierens das Schauen von Videos ist auch in doppelter Geschwindigkeit möglich -, die automatisierbare Überprüfung von Lösungen, die Nachverfolgbarkeit der Nutzung sowie die leichte Vervielfältigbarkeit. Auf der Sollseite können die hohen Produktionskosten zur Erstellung und sicherlich auch eine Vereinzelung von Lehrenden und Lernenden durch die indirekte Kommunikation über digitale Medien verbucht werden.

Bereits im Sommer 2019 und somit vor Auftreten der Corona-Pandemie hat das Wissenschaftsministerium Nordrhein-Westfalen (MKW) die Förderlinie OERContent.nrw zur Erstellung von offenen Lehrinhalten ausgeschrieben. Die geforderte allgemeine - offene - Nutzbarkeit von di- 


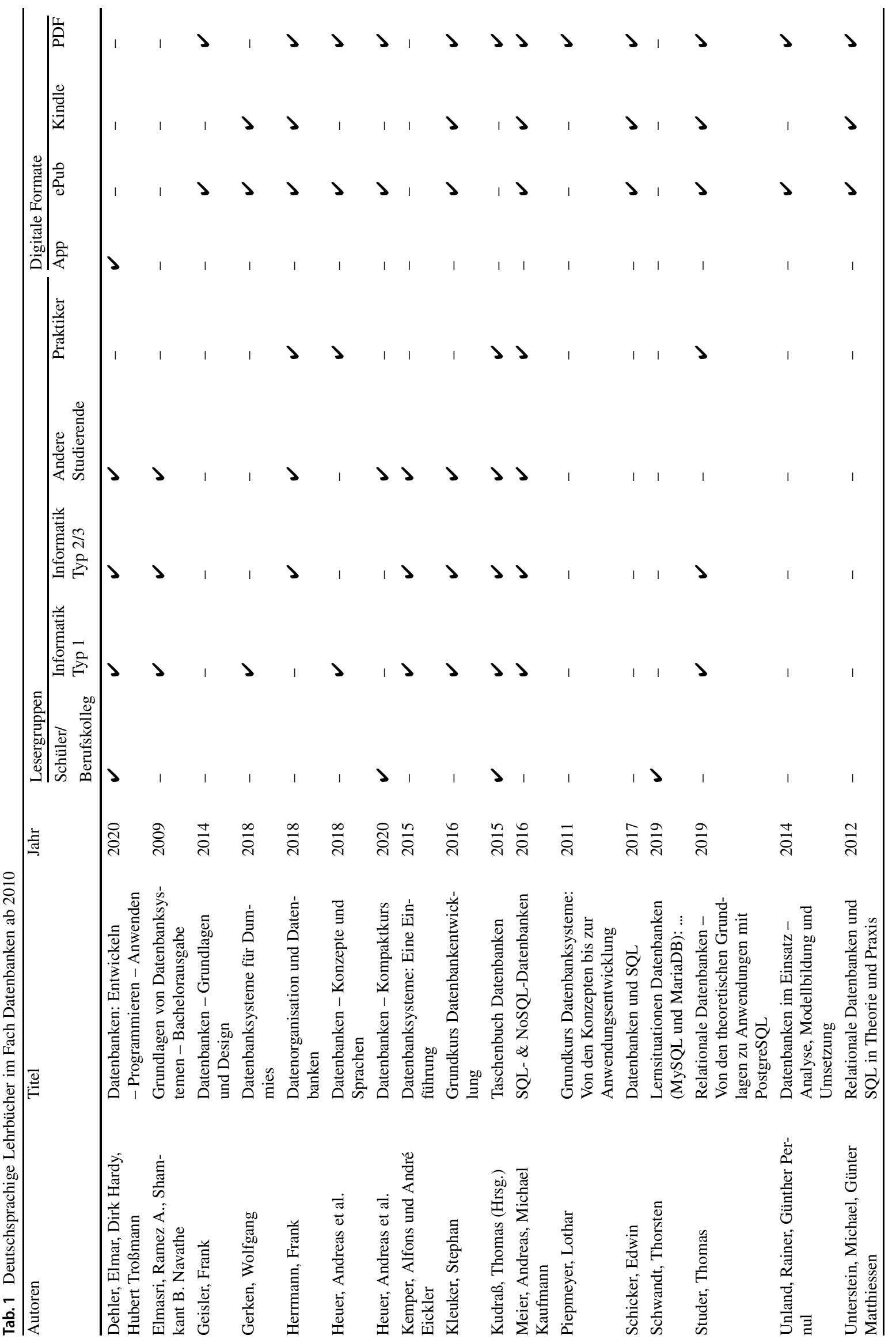




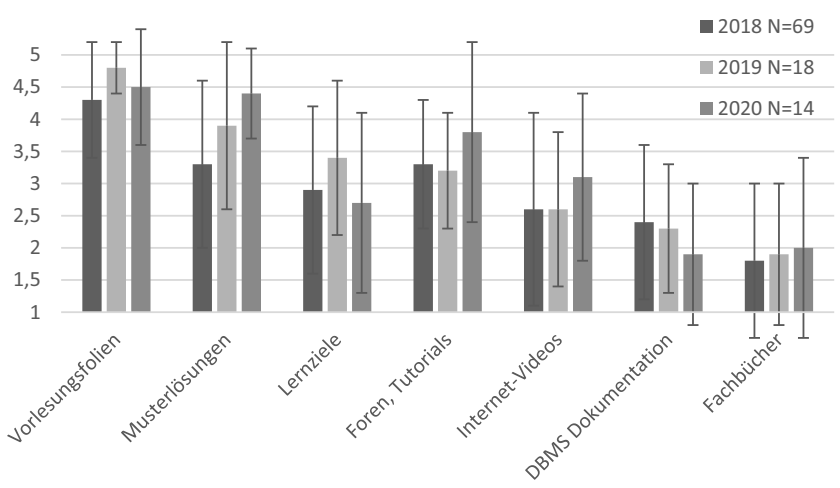

Abb. 1 Befragung zu der Mediennutzung unter Verwendung einer fünfwertigen Likert-Skala

gitalen Lerninhalten bzw. den Open Educational Resources (OER) ist seit etwa 2000 in der Diskussion [4, 5]. Weitere Bedingung zur Einreichung von Anträgen war die Bildung von Konsortien von mindestens drei Hochschulen für ,kleine" und mindestens sechs Hochschulen für „große“ Projekte.

Als eines von achtzehn Projekten der Förderlinie wird für zwei Jahre die Entwicklung von Inhalten für die Lehre im Fach Datenbanken in vielfältigen Lernszenarien (EILD.nrw) vom MWK gefördert. Konsorten sind die Hochschulen der Autoren dieses Beitrages.

Die Publikation von CC-lizensierten digitalen Lerninhalten (Open Educational Resources, OER) wirft einige Fragen auf:

- Inhalt: Welche Inhalte sollen für einen Studiengang vermittelt werden?

- Medium: Welches Format aus Vorlesungsaufzeichnung, Podcast, Erklärvideo und Animation erreicht die Zielgruppe?

- Granularität: Welchen Umfang sollen die Lerneinheiten für die Erklärung eines Fachbegriffs, eines Themas oder für eine Vorlesung haben?

- Interaktivität: Welcher Grad von Interaktivität ist möglich und kann auch zu hilfreichen Rückmeldungen an den Lernenden führen?

- Metadaten: Welche Beschreibungen helfen bei der Auswahl einer Lerneinheit?

- Fachbezug: Welche Besonderheiten sind für die Lehre in Informatik und im Fach Datenbanken vorhanden?

In diesem Artikel werden die Lehrbücher und Vorlesungsformate benannt, die aktuell für die digitale Lehre im Fach Datenbanken verfügbar sind. Anschließend folgen Erfahrungen mit dem Einsatz von Tools bzw. Plattformen sowie Ergebnisse aus Befragungen und Analysen von Zugriffen auf die Lernumgebungen der Autoren. Die Darstellung erfolgt auch unter Berücksichtigung der weitestgehend weggefallenen Präsenzzeiten im Sommersemester
2020, verursacht durch die Kontaktbeschränkungen während der Corona-Pandemie. Die hierbei gemachten Erfahrungen haben zu den nachfolgend dargestellten Anforderungen beigetragen, die eine gemeinsame Nutzung der vielfach aufwendig herzustellenden Lehr- und Lernmaterialien einfacher ermöglichen sollen. In Fazit und Ausblick dieses Beitrags folgt eine Reflektion, wie sich die Lehre zukünftig auch im Datenbankumfeld auf der Grundlage frei nutzbarer Lehreinheiten verändern kann. Auch diese Überlegungen wurden nicht zuletzt von den Erfahrungen durch das für viele Lehrende und Lernende zum Teil völlig andersartige Jahr 2020 geprägt.

\section{Digitale Lehre im Fach Datenbanken}

Der folgende Überblick berücksichtigt die Grundausbildung im Fachgebiet Datenbanken.

\subsection{Kompetenzen}

Kompetenzen auf dem Gebiet der Datenbanken gehören zum Pflichtbereich der Informatik. In den Empfehlungen zum Curriculum fordert die Gesellschaft für Informatik (GI) Kompetenzen „,nicht nur der theoretischen Grundlagen, sondern auch des Prozesses vom Datenbankentwurf bis zum Betrieb des datenbankgestützten Anwendungssystems sowie Datenanalyse und Grundlagen des maschinellen Lernens" [14].

Die GI definiert mit der Kerninformatik, der anwendungsorientierten Informatik und interdisziplinären Studiengängen mit einem wesentlichen Informatikanteil drei Typen von Studiengängen: grundständige Informatik (Typ 1), Informatik mit einem (starken) Anwendungsfach wie in der technischen Informatik, Medien- und Medizininformatik (Typ 2) und Informatik in einer interdisziplinären Ausrichtung wie der Wirtschaftsinformatik und Data Science (Typ 3). Für die Curricula aller drei Typen werden gleiche Kompetenzen im Bereich Datenbanken empfohlen, allerdings auch mit Abstrichen aufgrund des unterschiedlichen typbedingten Informatikanteils sowie unterschiedlichen Kompetenzstufen und Erweiterungen mit Anwendungen des jeweiligen Fachs [15-17, 19, 41]. In Master-Studiengängen werden spezielle Datenbankmodule angeboten, die sich von Hochschule zu Hochschule unterscheiden, und (bisher) nicht in Empfehlungen aufgenommen wurden.

\subsection{Lehre im Fach Datenbanken}

Die GI-Fachgruppe Datenbanken beschäftigte sich beim Herbsttreffen 2008 in Düsseldorf mit dem Thema „Quo Vadis: Formen der Datenbankausbildung und -weiterbildung“ 
Tab. 2 Anforderungen an den Austausch digitaler Lehrinhalte

\begin{tabular}{ll}
\hline Anforderung & Arten \\
\hline Lizensierung & Privat \\
& Ohne Lizenz \\
& Kommerziell \\
& Frei \\
Nachhaltigkeit & Nutzungserlaubnis \\
& Anpassbarkeit \\
& Technische Machbarkeit \\
& Datenschutz \\
& Stil, Layout, Notation \\
Zugänglichkeit & Sprache, Sprachniveau \\
& Berufliche Ausbildung \\
Kontextualisierung & Schule \\
& Hochschule \\
Inhaltsbeschreibung & Kenntnisse \\
& Lernziele \\
& Kompetenzen \\
Technische & Lernpfade \\
Anpassbarkeit & Änderbarkeit \\
\hline
\end{tabular}

[28]. Erst ein Jahrzehnt später erarbeiteten Teilnehmer auf einem Workshop der BTW 2019, wie die Lehre aktuell im Jahre 2019 im Fach Datenbanken aussieht und welche Erfahrungen damit gemacht wurden. Dabei wurden sowohl die Vorgehensweisen zum Lehren im Fach Datenbanksysteme - digitale Kommunikation, Portale, Blended Learning präsentiert als auch der Umgang aus der Lernerperspektive [30].

In der Informatik werden digitale Werkzeuge fachimmanent eingesetzt. Die Lehre selbst erfolgt seit langem mit diesen Tools - kommerziell, Open Source oder selbstentwickelt. Impliziert aber die Informatik im Allgemeinen keine praktische Arbeit, da alles am und mit dem Rechner möglich ist? - Nicht unbedingt, auch wenn sich ursprünglich analoge Arbeitsweisen wie beispielsweise das Pair-Programming auf digitale Formate abbilden lassen. Aber in Anwendungsbereichen wie zum Beispiel der Medientechnik mit der Nutzung von Foto- und Filmkameras und dem Green-Room oder in der Mediengestaltung auf und mit Papier wird weiterhin auch analog gearbeitet.

Gibt es Eigenheiten des Fachs Datenbanken bezüglich digitaler Lehre? - Die Verwendung von Datenbanken wird mittels der deskriptiven SQL-Programmierung gelehrt. Sie ist kompakt und erfordert anders als bei den meisten anderen Arten der Programmierung eine besondere Sichtweise. Im Curriculum wird die Programmierung meist vor der Verwaltung von Datenbanken behandelt. Die Vorgehensweise der prozeduralen Auswahl und Bearbeitung der Daten ist damit ,gewohnt“. Die Vorgehensweise deklarative und algebraisch definierte Verwaltung der relationalen Datenban-
Tab. 3 Lernmodule in EILD.nrw

\begin{tabular}{lll}
\hline Nr. & Thema & Niveau \\
\hline 1 & Konzepte von Datenbanksystemen & Bachelor \\
2 & Konzeptioneller Entwurf von Datenbanken & Bachelor \\
3 & Das relationale Datenmodell & Bachelor \\
4 & Programmierung mit SQL & Bachelor \\
5 & Entwicklung von Datenbankanwendungen & Bachelor \\
6 & Aufbau und Funktionsweise eines Daten- & Bachelor \\
7 & bankmanagementsystems & Bachelor/ \\
& NoSQL-Datenbanken & Master \\
8 & Verteilung von Datenbanken & Bachelor/ \\
& & Master \\
9 & Datenanalyse & Master \\
\hline
\end{tabular}

ken erscheint daher nicht nur anders, sondern auch ungewohnt.

\subsection{Lehrbücher}

In diesem Abschnitt gehen wir der Frage nach, welche Lehrbücher für Datenbanken derzeit auf dem Markt angeboten werden. Selbst wenn nur die grundlegenden in etwa den letzten zehn Jahren erschienenen Werke in deutscher Sprache - wie sie für die Lehre in Bachelor-Studiengängen gebräuchlich sind ${ }^{1}$ - einbezogen (siehe Abschn. 4) und rein SQL- und produktspezifische Werken ausgeschlossen werden, kommt man auf immerhin 17 Publikationen (Tab. 1). Die Lehrbücher lassen sich weiter nach Lesergruppen Schüler, Studierende und Praktiker unterscheiden. Insbesondere bei den Studiengängen wird zwischen Haupt- und Nebenfach sowie andere in Bezug auf Informatik unterschieden. Digital sind die meisten Lehrbücher verfügbar.

Weitergehende Möglichkeiten elektronischer Medien, beispielsweise eine Integration von interaktiven Elementen, werden durch die verwendeten Formate nicht genutzt. Ausnahme ist das Werk von Dehler et al., das als Anwendung auch Interaktionen ermöglichen soll [3]. Allerdings verfügen die Werke vielfach über digitales Begleitmaterial, welches über die Verlagswebsite oder von den Autoren bereitgestellt wird. Sie enthalten Vorlesungsfolien, Aufgaben, Skripte, Programmbeispiele und auch interaktive Datenbankzugänge zu den Datenbanken aus dem jeweiligen Lehrbuch. Wikis wie Wikipedia und das $e d b$-Wiki (s. Abschn. 3.2) beschreiben ergänzend zu Lehrbüchern die Fachbegriffe [10, 40].

\footnotetext{
1 Auch wenn allgemein das Sprachniveau B2 des GER (Gemeinsamer Europäischer Referenzrahmen) - selbständige Sprachverwendung mit der Fachhochschulreife in der ersten Fremdsprache erreicht werden kann, sollte doch aufgrund unterschiedlicher Bildungsbiografien der Studierenden in den ersten Semestern kein englisches Sprachverständnis vorausgesetzt werden.
} 


\subsection{Vorlesungsformate}

Vorlesungsformate werden von öffentlichen als auch kommerziellen Plattformen angeboten. Alfons Kemper bietet Vorlesungsaufnahmen auf seiner Webseite an [21]. Eine Vorlesungsaufzeichnung zum relationalen Modell ist von der U Frankfurt/Main (als Teil der Vorlesung „Logik und Datenbanken") verfügbar [36]. Auf der Plattform openHPI wird von Felix Naumann „Datenmanagement mit SQL“ als MOOC (Massive Open Online Course) angeboten [26, 27]. Bis zum Kursende 2013 hatten sich nach eigenen Angaben mehr als 7000 Teilnehmer registriert. In dem „eingefrorenen" Zustand ohne Aufgabenbewertung und Diskussionsrunden, aber mit Vortragsvideos und Aufgabenbearbeitung, haben sich über 12.000 weitere Teilnehmer registriert (November 2020).

Beim Inverted oder auch Flipped Claasroom werden die zentralen Aktivitäten des Lehrens und Lernens umgekehrt. Die Inhaltsvermittlung und -erschließung erfolgt weniger traditionell im Rahmen einer gemeinsamen Präsenzphase, sondern orts- und zeitungebunden über das Internet. Die nachgeschaltete Präsenzphase dient zur Vertiefung, Übung oder Diskussion des Gelernten [18]. Zu den Pionieren speziell im Umfeld der Datenbanken gehört Jens Dittrich [6, 7]. Er beschreibt sein Vorlesungsformat als ein hybrides Format zwischen traditioneller Vorlesung und den MOOCs.

In kommerziellen Plattformen werden viele weitere Inhalte angeboten. So sind in Lynda.com zum Thema Datenbanken nach eigenen Angaben 115 Kurse mit 4657 Video Tutorials verfügbar (26.10.2019). SpringerLink bietet von Alan Taylor Hörclips von bis zu 10 min Länge über ,,SQL and Relational Databases" an [37].

In YouTube reißt die Liste von Treffern zur Suche von deutschsprachigen Erklärvideos zum Suchbegriff „Datenbanken“ nicht ab. Allerdings fehlen hier eine strukturierte Zusammenstellung und Kennzeichnung von Lernzielen, um ein „Lost in Hyperspace“ zu vermeiden. Hinzu kommt, dass interaktive Elemente zur Aktivierung der Lerninhalte in den Videos fehlen.

An der FH Dortmund wurden zahlreiche Lernanwendungen sowie Lehrmodule mit integrierten Lehrvideos entwickelt [32-34]. In einer Studie wurde das Lernverhalten aus Sicht der Studierenden erhoben. In drei aufeinanderfolgenden Jahren von 2018 bis 2020 wurden die Studierenden des dritten Fachsemesters in den Studiengängen Allgemeine Informatik befragt. Es hat sich gezeigt, dass in der Lernplattform bereitgestellte Unterlagen (Vorlesungsfolien, Musterlösungen) eine sehr hohe Akzeptanz bei den Studierenden besitzen. Eine mittlere Akzeptanz erfahren die als Reflexionsfragen formulierte Lernziele sowie Internetquellen, wie Foren, Tutorien und Internet-Videos. Eine geringere Relevanz haben dabei die Datenbankdokumentation der Hersteller und einschlägige Fachbücher. Insgesamt zeigt sich, dass viele Studierende sich auf die von dem Lehrenden bereitgestellten Lernmedien fokussieren und weniger weitere Angebote nutzen (Abb. 1).

Ähnliche Erfahrungen hinsichtlich der Nutzung elektronisch bereit gestellter Lehr- und Lernmedien wurden auch an der Hochschule Bonn-Rhein-Sieg gemacht. Allerdings werden die einführenden Datenbankvorlesungen dort in Anlehnung an den „Inverted Classroom“ durchgeführt, wodurch die als Reflexionsfragen formulierten Lernziele als Mittel des aktiven und eigenständigen semesterbegleitenden Lernens wesentlich an Bedeutung gewinnen. Hier wurde in Evaluierungen eine deutliche Polarisierung des Studierendenverhaltens festgestellt: während die eine Gruppe das eigenständige Lernen und Experimentieren im Vorfeld der Präsenzphase ausdrücklich lobte, zeigte die andere Gruppe deutliche Schwierigkeiten im aktiven Studierverhalten und zog die eher passive „konsumorientierte“ traditionelle Form der Vermittlung von Lehr- und Lerninhalten vor.

\section{Nutzung von Tools zur Lernunterstützung}

Praktika sind in der Regel ein integraler Bestandteil von Datenbanklehrveranstaltungen, in denen die Interaktion mit einem Datenbankmanagementsystem trainiert wird. Ergänzend zu Präsenzpraktika bietet sich eine Einbindung von Lerntrainern zum Selbststudium an. Der folgende Abschnitt beschreibt unsere Erfahrungen in der Einbindung bzw. Entwicklung von Lerntrainern und der Entwicklung einer Lernplattform.

\subsection{Einbindung von Lerntrainern}

In zahlreichen Tools werden B- und auch B+-Bäume visualisiert, so durch David Galles [13], der seine Website und auch die Quellen frei zur Verfügung stellt. Hier ist ein Nachvollziehen der Operationen am Index möglich, aber eine selbständige Ausführung mit einem besseren Lernerfolg wird nicht unterstützt.

Im Umfeld der Datenbank-Optimierung wurde an der HS Bonn-Rhein-Sieg eine Lernplattform weiterentwickelt, die mittels Visualisierungen die Auswirkungen von ausgewählten Tuningmaßnahmen anschaulich macht [38].

Das Tool Umlet+RTE der HS Düsseldorf ermöglicht die Modellierung von relationalen Datenbanken mit UML im Round-Trip-Engineering [35]. Die Einsetzbarkeit wird aber durch die Nichtpassung des frei verfügbaren UMLEditors UMlet zur Erkennung der Zeichnungselemente als UMLKomponenten erschwert.

Zur Ausführung relationaler Ausdrücke kann das Tool RelaX auf einer Website der Universität Innsbruck verwendet und über Github ausgeführt werden [22, 31]. Da der Source Code in Github veröffentlicht ist, kann er in dieser 
Umgebung genutzt oder im eigenen LMS integriert werden. Die vorhandenen Schemata können durch eigene erweitert werden. Die Integration in Moodle und Erweiterbarkeit mit eigenem Schema konnte an der HS Düsseldorf problemlos umgesetzt werden. Allerdings musste die verwendete Notationen in den eigenen Materialien angepasst werden.

\subsection{Entwicklung der edb}

An der TH Köln wird seit 2003 das E-Learning Datenbank Portal (edb) mit zahlreichen Tools, einem Wiki sowie einer Datenbank mit über 1700 Fragen, Antworten und Erklärungen entwickelt und in der Lehre eingesetzt [8-11].

Im Multiple Choice Test (MCT) wurden im Laufe der Jahre 1417 Multiple-Choice-Fragen (Stichtag: 27.09.2020) gesammelt. $\mathrm{Zu}$ jeder Frage gibt es auch Erklärungen und ein Feedback-Formular. In einem Lernmodus werden nur Fragen präsentiert, die der Lernende noch nicht richtig beantwortet hat.

Im 3NF-Normalformentrainer werden mittels Ausgangstabelle und einer textuellen Beschreibung die funktionalen Abhängigkeiten vorgegeben. Der Benutzer kann dann mittels Drag\&Drop einen Primärschlüssel bestimmen, den Typ der Abhängigkeiten festlegen (voll, partiell oder transitiv) und daraus die 2. Normalform und die 3 . Normalform ableiten.

Der SQL-Trainer enthält verschiedene Datenbankschemata und insgesamt ca. 300 vorformulierte Anfragen mit unterschiedlichem Schwierigkeitsgrad, die sich mittels einer SELECT-Anweisung in edb interaktiv beantwortet lassen. Diese SQL-Anfragen werden online an eine Datenbank geleitet und dort ausgeführt.

Im PL/SQL-Trainer kann der Benutzer interaktiv Datenbanktrigger, Prozeduren und Funktionen nach OracleSyntax und festgelegten Aufgabenstellungen erstellen und in der Datenbank online ausführen lassen. Falls die Syntax korrekt ist, wird das Ergebnis des Triggers mit einem Beispiel-DML-Befehl als Prüfstatement bzw. direkt aus Rückgabewert der Funktion oder der Prozedur mit der Musterlösung verglichen und bewertet. Um die Mehrbenutzerfähigkeit zu gewährleisten, wird für jeden PL/SQL-Nutzer ein eigenes Datenbankschema generiert und nach dem Abmelden aus $e d b$ wieder gelöscht.

Ein Wiki, ein ER-Trainer sowie ein interaktives SQLPuzzle zum Legen von Assoziationen für Datenbankbegriffe [28] runden das Angebot von edb ab.

\subsection{Evaluierung der edb}

Im Rahmen einer studentischen Projektarbeit wurde in [2] die Nutzung von edb untersucht. An einer Befragung beteiligten sich insgesamt 82 Nutzer vorwiegend von der TH Köln. Die edb-Nutzer finden die Anwendung insgesamt sehr hilfreich (22\%), hilfreich (49\%), ausreichend hilfreich (19\%) und überhaupt nicht hilfreich (10\%). Bei den Tools wird der SQL-Trainer am häufigsten genutzt $(68 \%)$, gefolgt vom MCT $(66 \%)$, dem Normalformentrainer $(52 \%)$, dem PL/SQL-Trainer (38\%), während das SQL-Puzzle (14\%) und das DB-Wiki (22\%) weniger Zuspruch fanden.

Von studentischer Seite wurden in der edb insbesondere im SQL-Trainer mehr hilfreiche Tipps gewünscht, die über die Datenbankfehlermeldungen hinausgehen. Hierzu wurden seit März 2019 alle fehlerhaften SQL-Anweisungen mitprotokolliert und mit der gespeicherten Musterlösung verglichen [11]. Daraus wurde manuell ein Entscheidungsbaum generiert und auch schon in edb implementiert, der die Benutzerfehler klassifiziert und automatisch Hinweise zur Verbesserung der Aufgabe ausgibt.

Andere Hochschulen wurden zur Nutzung der von der TH Köln gehostete edb-Plattform eingeladen. Obwohl das Angebot von den jeweiligen Lehrenden den Studierenden empfohlen wurde, wurde die Plattform bisher nur wenig von den Studierenden der anderen Hochschulen genutzt.

\subsection{Digitale Lehre in Corona-Zeiten}

Im Rahmen der studentischen Projektarbeit [2] wurde untersucht, wie das edb-Portal gerade in Corona-Zeiten genutzt wurde. Die Besucherhäufigkeit hat sich seit März 2020 stark erhöht. Für den SQL Trainer wurde eine Protokolltabelle angelegt, in der alle fehlerhaften Statements der edb-Nutzer gespeichert wurden, um daraus Tipps zur Verbesserung im Vergleich mit der Musterlösung abzuleiten. Im Vergleich zu den Monaten vom September 2019 bis zu März 2020 verdoppelten sich die Zugriffszahlen ab April bis September 2020. Deutlich lassen sich auch an höheren Zugriffzahlen Klausurzeiten erkennen.

Eine Studierendenbefragung an der HS Düsseldorf hat die Umstellung von Präsenzlehre mit digitalen Formaten auf eine rein online basierte Lehre durchaus mit Vorteilen verbunden [1]. Die meisten Befragten schätzen die räumliche und zeitliche Flexibilität (74\%), die Familienfreundlichkeit (53\%) und das hohe Angebot an Lernmedien $(58 \%)$. Es scheint sich aber auch die Meinung durchzusetzen, dass Online-Lehre unter mehr Ablenkung leidet (62\%). Die Studierenden sind sich der außergewöhnlichen Umstände im Sommersemester 2020 durchaus bewusst. Zwar werden etwaige Probleme deutlich angesprochen und offen kritisiert, dennoch wird in der Globalbewertung der kurzfristigen Umstellung fast ausschließlich auf digitale Lehre „angemessen Rechnung getragen“.

Erste Erfahrungen mit E-Klausuren liegen an der TH Köln vor. Wegen der hohen zu erwartenden Teilnehmerzahlen - im September 2020 wurden insgesamt 470 Teilnehmer in den beiden Datenbankklausuren DBS1 und DBS2 registriert - waren Präsenzklausuren in Corona Zeiten dort nicht 
möglich. Es wurden auf Basis von edb und Vorlesungsunterlagen E-Klausuren mit Hilfe des Lernmanagementsystems ILIAS erstellt. Die Übertragung der Aufgabenstellung in das Home-Office der Studierenden selber war unproblematisch. Allerdings verführen derartige Klausuren zu gemeinschaftlichen Täuschungsversuchen, da sich nicht überprüfen lässt, wer sich noch im gleichen Raum befindet. Teilweise waren diese Versuche sehr plump und einfach nachweisbar. Auch die Art der Lösung der Aufgaben hat sich verändert, da oft nur nach ähnlichen Lösungen im Internet gesucht wird. Für die nächsten e-Klausuren wurde daher mehr Varianz eingebaut, so dass jeder Prüfling eine andere vergleichbare Klausur erhält. Insgesamt erachtet die TH Köln den Rückweg zur Präsenzklausur als sinnvoll, aber auch die Nutzung der Lernmethoden als Blended Learning. Digitale Lehre soll nicht den persönlichen Kontakt in einer Präsenzveranstaltung und auch nicht eine Vorort-Klausur ersetzen.

\section{Anforderungen an die Publikation von digitalen Lerneinheiten}

Bei der Evaluation der Tools fällt auf, dass Tools gerne von Studierenden angenommen werden, wenn sie inhaltlich nahe beim Vorlesungsstoff sind. Daher sollten die Lehrenden beispielsweise ihre eigenen Datenbankschemata verwenden können. Dozenten begrüßen die Möglichkeit, in einer Administrationsumgebung aus vorhandenen Aufgabenpools auszuwählen und bei Bedarf auch selber diese Aufgaben anzupassen. Die Präsenzlehre wird explizit nur von wenigen Tools unterstützt [20, 29].

Offenbar ist ein Austausch von digitalen Lehrmaterialien zwischen Lehrenden in Datenbankveranstaltungen und deren direkte Einbindung in die eigene Lehre nicht immer einfach möglich. In diesem Kapitel wird der Frage nachgegangen, welche Eigenschaften Lehr- und Lernmaterialien erfüllen sollen, damit sich diese möglichst einfach in die vielfältigen Lernszenarien des Fachs Datenbanken und intendierten Lehrmethoden - wie Inverted Classroom, Just in Time Teaching und Peer Instruction - einbinden lassen. Die hieraus abgeleiteten Anforderungen resultieren u.a. aus den im letzten Kapitel beschriebenen Erfahrungen.

Die traditionelle Form des Austauschs ist eine Nutzung von Lehrbüchern und deren Begleitmaterial in Lehrveranstaltungen. Einschränkungen der Nutzbarkeit ergeben sich aus den Lizenzen, unter denen die Lehrmaterialien veröffentlicht werden. So kann eine Lizensierung von digitalen Lehrbüchern nicht erfolgen, wenn die benötigten finanziellen Mittel das Budget der Bibliotheken übersteigen. Auch wird der Zugriff auf die Lehrbücher durch manche Verlage eingeschränkt - zeitlich befristet, gleichzeitige Nutzer -, so dass eine kontinuierliche Bereitstellung für die Studierenden teilweise nicht gegeben ist.

Entsprechende Einschränkungen gibt es auch bei frei verfügbaren digitalen Lernmedien. Die Einbindung in die eigene Umgebung ist nicht immer möglich, wenn die Erlaubnis zur Nutzung, die Anpassbarkeit oder die technische Machbarkeit fehlen. Bei der Nutzung in einer „fremden“ Umgebung fehlt die Nachhaltigkeit dieser Angebote, wenn diese durch die jeweiligen Anbieter jederzeit geändert oder entfernt werden können. Auch der Datenschutz muss gewährleistet werden können.

Hinzu können Einschränkungen hinsichtlich der Zugänglichkeit der Lernmaterialien für die Studierende kommen. Lehrbücher und Lernmaterialien unterscheiden sich in Stil, Layout, Form sowie in den verwendeten Notationen und Sprachen. Für die Aufnahme englischsprachiger Studiengänge wird meist das Niveau B2 des Gemeinsamen Europäischen Referenzrahmens gefordert, das etwa nach fünf Schuljahren bereits durch die Fachhochschulreife erreicht werden kann [23]. Die benötigte Sprachkompetenz zum Verstehen englischer Fachliteratur dürfte aber erst im Verlauf des Studiums aufgebaut werden können. Insbesondere für einführende Datenbank-Lehrveranstaltungen in den ersten Semestern eines deutschsprachigen Bachelorstudiums werden daher vorrangig deutschsprachige Lehrbücher eingesetzt, um sprachliche Hürden zu vermeiden.

Inhaltlich ergeben sich Einschränkungen hinsichtlich der Kontextualisierung von Lernmedien. Die Materialien unterscheiden sich in ihrer Orientierung für beruflicher Ausbildung oder der Praxis, Schule oder Hochschule (vgl. Tab. 1). Verschiedenste relationale Beispielanwendungen werden - in Lehrbüchern auch durchgängig - verwendet, aber nicht alle Beispiele sind für jeden Studiengang geeignet. Hinzu kommt eine Verwendung von unterschiedlichen Nomenklaturen in den Lernmedien, beispielsweise zur Darstellung von relationalen Modellen. Die Lerneinheiten sollen sich in den unterschiedlichen Studienfächern der Informatik - Angewandte Informatik, Medizininformatik, Wirtschaftsinformatik, Medieninformatik u.a. sowie in den unterschiedlichen Studiengangsstufen bzw. -typen Bachelor, Master, Dual - zuordnen lassen.

Oftmals ist unklar, welche Kenntnisse bei den Lernenden vorausgesetzt werden. Beispielsweise kann nicht einfach unterschieden werden, ob jeweils zwei gefundene Lernmedien identische Sachverhalte auf eine andere Art und Weise darstellen oder aufeinander aufbauen. Daher ist durch den Lehrenden eine Auswahl zu treffen, so dass Lernern identische Sachverhalte nicht doppelt und in einer ungeeigneten Reihenfolge angeboten werden - oder hier gar Widersprüche auftreten, was „normal“ ist, aber den Lernenden verwirrt.

Die Definition der zugehörenden Lernpfade hat zum Ziel, dass ein „Lost in Hyperspace“ der Lernenden vermie- 
den wird. Der Aufbau der Lernpfade erfolgt typischerweise auf der Lernplattform der Hochschule.

Einschränkungen bei der Integration der Lernmedien treten oft dadurch auf, dass bestimmte Plattformen vorausgesetzt werden, in denen die Anwendungen programmiert werden können wie den verwendeten Produkten für Datenbankmanagementsysteme oder sich auf hochschulspezifische Infrastrukturen wie beispielsweise Serveradressen und Installationspfaden bezogen wird. Die Lerneinheiten sollen sich in verschiedenen Systemumgebungen aus dem kommerziellen und Open Source-Bereich sowie in den Lernplattformen wie Moodle oder ILIAS einsetzen lassen.

Die Formen der Lizensierung, Nachhaltigkeit und $\mathrm{Zu}-$ gänglichkeit, die Möglichkeiten zur Kontextualisierung und die inhaltliche wie technische Anpassbarkeit sowie bei Nutzung von Plattformen die Einhaltung des Datenschutzes sind die bestimmenden Faktoren zum Austausch digitaler Inhalte in der Lehre (Tab. 2).

\section{Das EILD-Konzept}

Als konstruktive Antwort auf die geschilderte Herausforderung wird im Folgenden das Konzept zur Entwicklung von Inhalten für die Lehre im Fach Datenbanken in vielfältigen Lernszenarien (EILD) vorgestellt, dessen Umsetzung von der Digitalen Hochschule des Landes Nordrhein-Westfalen (DH.NRW) gefördert wird und im September 2020 von den Autoren begonnen wurde.

In EILD.nrw wird ein Konzept mit weitgehender Adaptierbarkeit und Wiederverwendbarkeit der Lehr- und Lerninhalte umgesetzt. Die Abdeckung der Themen von Grundvorlesungen im Fach Datenbanken soll in sieben Lernmodulen erfolgen. Zwei auf den Grundlagen aufbauende Module orientieren sich am Master-Niveau. Die Trennung von didaktischem Konzept und Anwendungsbereichen aus den jeweiligen Studiengängen mit der Angabe von Metadaten und Best-Practice Szenarien ermöglicht eine eigenständige Nutzung von Lerneinheiten kleiner Granularität. Die Lehrenden können diese Lerneinheiten auf die jeweiligen Kontexte und die intendierte Lehrmethode - wie Inverted Classroom, Just in Time Teaching und Peer Instruction - anpassen. Bei den Lerneinheiten handelt es sich um Video-Sequenzen aus aufgezeichneten Lehrveranstaltungen, Tutorien zum Erlernen von Techniken und speziellen Werkzeugen, Animationen zur Verdeutlichung komplexer Abläufe, Online-Tests und Quizze zum Feststellen der Lernfortschritte sowie Experimentierumgebungen speziell zum Vertiefen der Programmierung mit SQL. Die Lernmodule des Projekts werden in zwei Iterationen in den Studiengängen der Konsorten und für die Digitale Hochschule NRW eingesetzt und so unter Einbeziehung der Studierenden evaluiert. In der ersten Ite- ration werden etwa 1000 Studierende der Hochschulen des Konsortiums beteiligt.

Die in den Lernmodulen des Projekts (Tab. 3) vermittelten Kompetenzen beinhalten alle von der GI empfohlenen Kompetenzen im Fach Datenbanken und Informationssysteme für Bachelorstudiengänge [14]. Diese können wie in einem Baukastensystem anhand eines Leitfadens für die Lehrenden je nach Hochschule (Dozent) und Typ des Studiengangs ausgewählt und adaptiert werden. In sechs Lernmodulen erfolgt eine Abdeckung der Themen von Grundvorlesungen im Fach Datenbanken. Ein weiteres Lernmodul zum Thema der NoSQL-Datenbanken erweitert die Inhalte um zum relationalen Modell alternative Entwicklungen [25]. Bei den beiden auf den Grundlagen aufbauenden Lernmodulen zu Master-Veranstaltungen handelt es sich um Verteilte Datenbanken und Datenanalyse. Die vermittelten Kompetenzen sind in den Empfehlungen der GI [14] sowie auch den Studienordnungen bzw. Modulbeschreibungen in Studiengängen der Hochschulen beschrieben [19].

Die Interoperabilität der Lerneinheiten mit verschiedenen Lern-Management Systemen (LMS) wird durch die Verwendung von gebräuchlichen Schnittstellen und Austauschformaten gewährleistet. Zum Austausch von Lernmodulen und Lerntests werden das Sharable Content Object Reference Model (SCORM) und das Question \& Test Interoperability (QTI) Austauschformat verwendet. Zur Anbindung der Aufgabentrainer kann die Learning Tools Interoperability (LTI) Schnittstelle der Lern-Management Systeme genutzt werden.

\section{Fazit und Ausblick}

Auch aus den Erfahrungen mit der Umstellung auf (fast) $100 \%$-Online-Lehre während des (ersten) „Corona“-Semesters im Sommersemester 2020 wurde deutlich, dass es nicht nur ein Konzept für eine erfolgreiche Lehre geben kann, sondern vielmehr ein Zusammenspiel aus mehreren Einflussfaktoren über den Erfolg entscheiden wird: ${ }^{2}$

- eine Mischung aus synchronen und asynchronen digitalen Medien,

- eine nachhaltige Lizensierung,

- ein klares didaktisches Konzept,

- eine niederschwellige Einbindung der Studierenden durch Melden, Voting, anonyme Fragebögen (Kummerkasten), Breakout- und Fragen-Sessions usw.,

- der Absprache unter den Lehrenden eines Studiengangs zur Eingrenzung der Tool- und Methodenvielfalt,

- der Verwendung interaktiver Tools.

2 Danke für ihre Beiträge an die Teilnehmer auf dem Workshop „Medieninformatik 2020 - Lehren im neuen Jahrzehnt“ auf der MuC 2020. 
Ein Mix aus digitalen Lehrbüchern, eigenen und OER Lehr- und Lerneinheiten wird diesen Ansprüchen wahrscheinlich am ehesten gerecht. In diesem Zusammenhang sollen kommerzielle Lehrbücher und professionell entwickelte E-Learnings nicht ausgeschlossen werden, sofern sich das nachhaltig und budgetierbar realisieren lässt. OER werden eine weitergehende Akzeptanz bei Lehrenden und Lernenden finden, wenn der Nutzen den Aufwand bei der Erstellung und Verwendung übersteigt.

Funding Open Access funding enabled and organized by Projekt DEAL.

Open Access Dieser Artikel wird unter der Creative Commons Namensnennung 4.0 International Lizenz veröffentlicht, welche die Nutzung, Vervielfältigung, Bearbeitung, Verbreitung und Wiedergabe in jeglichem Medium und Format erlaubt, sofern Sie den/die ursprünglichen Autor(en) und die Quelle ordnungsgemäß nennen, einen Link zur Creative Commons Lizenz beifügen und angeben, ob Änderungen vorgenommen wurden.

Die in diesem Artikel enthaltenen Bilder und sonstiges Drittmaterial unterliegen ebenfalls der genannten Creative Commons Lizenz, sofern sich aus der Abbildungslegende nichts anderes ergibt. Sofern das betreffende Material nicht unter der genannten Creative Commons Lizenz steht und die betreffende Handlung nicht nach gesetzlichen Vorschriften erlaubt ist, ist für die oben aufgeführten Weiterverwendungen des Materials die Einwilligung des jeweiligen Rechteinhabers einzuholen.

Weitere Details zur Lizenz entnehmen Sie bitte der Lizenzinformation auf http://creativecommons.org/licenses/by/4.0/deed.de.

\section{Literatur}

1. Bluszcz-Weinem O et al (2020) Studieren in Zeiten von Corona, Studierendenbefragung im Sommersemester 2020. HS Düsseldorf, Düsseldorf (Kurzbericht)

2. Boz S (2020) Analyse und Bewertung der Web-Applikation „Das eLearning Datenbank Portal edb“. TH Köln, Köln (studentische Projektarbeit)

3. Dehler E, Hardy D, Troßmann H (2020) Datenbanken: Entwickeln - Programmieren - Anwenden, 4. Aufl. Europa-Lehrmittel, Haan

4. Deimann M (2018) OER-Forschung - Warum es sie bisher nicht gab und wie sich das ändern kann. Synergie 5:70-75

5. Deutscher Bildungsserver (2016) Machbarkeitsstudie zum Aufbau und Betrieb von OER-Infrastrukturen in der Bildung (Stand: Februar 2016)

6. Dittrich J (2014) Die Umgedrehte Vorlesung - Chancen für die Informatiklehre. Datenbank Spektrum 14:69-76

7. Dittrich J Datenbanklernen.de. Zugegriffen: 4. Dez. 2020

8. Faeskorn-Woyke H, Bertelsmeier B EDB - Das eLearning Datenbank Portal. https://edb2.gm.th-koeln.de. Zugegriffen: 5. Jan. 2021

9. Faeskorn-Woyke H, Bertelsmeier B, Riemer P, Bauer E (2007) Datenbanksysteme - Theorie und Praxis mit SQL2003, Oracle und MySQL. Pearson, München

10. Faeskorn-Woyke H, Bertelsmeier B, Gawenda D, Kasper A (2013) Erfahrungen mit dem kooperativen E-Learning-Datenbankportal edb. In: Breiter A, Rensing C (Hrsg) DeLFI 2013. Gesellschaft für Informatik, Bonn, S 285-288

11. Faeskorn-Woyke H, Bertelsmeier B, Strohschein J (2020) A decision tree approach for the classification of mistakes of students learning SQL, a case study about SELECT statements. In: 18th DELFI Conf 09.2020
12. Fakultätentag Informatik e.V. (FTI) (2020) Positionspapier „Digitalisierung der Hochschullehre“ (27. März). https://www.ftinformatik.de. Zugegriffen: 05.01.2021

13. Galles D Data structure visualization. https://www.cs.usfca.edu/ $\sim$ galles/visualization/. Zugegriffen: 29. Sept. 2020

14. Zukunft O (2016) Empfehlungen für Bachelor- und Masterprogramme im Studienfach Informatik an Hochschulen. Gesellschaft für Informatik e. V., Bonn

15. Jung R (2017) Rahmenempfehlung für die Ausbildung in Wirtschaftsinformatik an Hochschulen. Gesellschaft für Informatik e. V., Bonn

16. Maehle E (2018) Curriculum für Bachelor- und Masterstudiengänge Technische Informatik. Gesellschaft für Informatik e. V., Bonn

17. GI e. V. (2018) Data Literacy und Data Science Education: Digitale Kompetenzen in der Hochschulausbildung

18. Handke J, Sperl A (Hrsg) (2012) Das Inverted Classroom Model 1. ICM-Konferenz, Begleitband. Oldenbourg, München

19. Heinecke AM, Kindsmüller MC, Noss C, Rakow TC, Rumpler M, Wolters C (2017) Medieninformatik 2017: Berufsbilder, Färbungen, Curricula und Erfahrungen. In: Burghardt M, Wimmer R, Wolff C, Womser-Hacker C (Hrsg) Mensch und Computer 2017 Workshopband. Gesellschaft für Informatik e. V., Regensburg

20. Joeres S, Rakow TC (2013) Interaktives Projektrepository zur Unterstützung der Kollaboration in Präsenzumgebungen. In: Reiter A, Rensing C (Hrsg) DeLFI 2013, S 289-292 (LNI(P-218))

21. Kemper A et al. Vorlesungsaufzeichnungen zu Datenbanksysteme: Eine Einführung. https://db.in.tum.de/teaching/bookDBMSeinf/ aufzeichnungen/?lang=de. Zugegriffen: 4. Dez. 2020

22. Kessler J, Tschuggnall M, Specht G (2019) RelaX: a webbased execution and learning tool for relational algebra. In: Grust $\mathrm{T}$ et al (Hrsg) BTW 2019. Gesellschaft für Informatik, Bonn, S 503-506

23. Keuck S, Wahlers M (2018) HRK-EXPERTISE-Manual: Mehrsprachigkeit in Studium und Lehre. HRK, Hochschulrektorenkonferenz, Berlin

24. Kultusministerkonferenz (2019) Empfehlungen zur Digitalisierung in der Hochschullehre. https://www.kmk.org/fileadmin/Dateien/ pdf/PresseUndAktuelles/2019/BS_190314_Empfehlungen_Digita lisierung_Hochschullehre.pdf. Zugegriffen: 14. März 2019

25. Kudraß T (2015) Taschenbuch Datenbanken, 2. Aufl. Hanser, München

26. Naumann F, Jenders M, Papenbrock T (2014) Ein Datenbankkurs mit 6000 Teilnehmern. Informatik Spektrum 37:333-340

27. Naumann F (2020) Datenmanagement mit SQL. https://open.hpi. de/courses/sql. Zugegriffen: 4. Dez. 2020

28. Rakow TC, Faeskorn-Woyke H, Schiefer B, Vossen V, Wäsch J (2009) Tools für die Lehre im Fach Datenbanken. Datenbank Spektrum 9(29):5-13

29. Rakow TC, Korezki V, Bender A (2012) Comparing the contrast for electronic and traditional whiteboards in a real-life environment. In: Proc. Electronic Displays Conference Nuremberg, Germany

30. Rakow TC, Faeskorn-Woyke H (2019) Digitale Lehre im Fach Datenbanken. In: Meyer H, Ritter N, Thor A, Nicklas D, Heuer A, Klettke M (Hrsg) BTW 2019 - Workshopband. Gesellschaft für Informatik, Bonn, S 97-98

31. RelaX - relational algebra calculator. Github.io. Zugegriffen: 4. Dez. 2020

32. Saatz I (2013) Linking mobile learning and offline interaction. In: Proceedings of the 2013 ACM conference on Pervasive and ubiquitous computing adjunct publication UbiComp '13 Adjunct. ACM, Zürich, S 1389-1392

33. Saatz I, Kienle A (2013) Learning with E-Flashcards-Does It Matter? In: Hernández-Leo D, Ley T, Klamma R, Harrer A (Hrsg) Scaling up Learning for Sustained Impact EC-TEL 2013. LNCS, Bd. 8095. Springer, Berlin 
34. Saatz I (2017) Wo steckt nur der Fehler in der SQL-Anfrage? Semantische Prüfung von Lösungen. In: Proc. Third Workshop „Automatische Bewertung von Programmieraufgaben" ABP.

35. Salgert B, Rakow TC (2017) Modellierung von relationalen Datenbanken mit UML im Round-Trip-Engineering. In: LNI, 17. BTW 03.2017, S 355-364 (Workshopband)

36. Schweickardt N (2012) Das Relationale Modell. In: Logik und Datenbanken. Goethe-Universität, Frankfurt/M. http://electure. studiumdigitale.uni-frankfurt.de/index.php?cat $=1 \&$ sem $=6 \&$ video list=317. Zugegriffen: 30 . Nov. 2020

37. Taylor A (2019) Introducing SQL and relational databases. Apress, New York

38. Thieme M, Zinner N, Knolle H (2009) Eine Lernplattform zur Visualisierung der Auswirkungen von Datenbank-Optimierungen. In: DOAG
39. Wannemacher K et al (2016) Digitale Lernszenarien im Hochschulbereich. Arbeitspapier Nr. 15. Hochschulforum Digitalisierung, Berlin

40. Wikimedia Foundation Inc. Wikipedia, Die freie Enzyklopädie. https://de.wikipedia.org/. Zugegriffen: 30. Nov. 2020

41. Wolters C, Kindsmüller MC, Heinecke AM, Rakow TC, Dahm M, Jent S, Rumpler M (2019) Medieninformatik 2019: Kompetenzorientierte Lehr-Lernszenarien in der Medieninformatik. In: Mensch und Computer 2019 - Workshopband. Gesellschaft für Informatik e. V., Bonn 